\title{
peb gegen den Sitzenden Lebensstil
}

\author{
A. Lambeck ${ }^{1}$
}

\section{Zusammenfassung}

Die Plattform Ernährung und Bewegung e.V. (peb) engagiert sich in vielfältiger Weise für die Gesundheitsförderung und gegen den Sitzenden Lebensstil von Kindern und Jugendlichen. Da der Sitzende Lebensstil als eigenständiger Risikofaktor für die Gesundheit von Kindern bisher vernachlässigt wurde, erarbeitete peb zunächst die inhaltlichen Grundlagen zum Thema. Daraufhin wurden entsprechende Instrumente zur Ansprache von Multiplikatoren und Verbrauchern entwickelt und umgesetzt.

Stichworte: Sitzender Lebensstil, Sitz-Check, Peb \& Pebber, Plattform Ernährung und Bewegung

Die Plattform Ernährung und Bewegung e.V. (peb) engagiert sich zur Prävention von Übergewicht bei Kindern und Jugendlichen für einen gesunden Lebensstil mit ausgewogener Ernährung und viel Bewegung. Dabei geht peb ursachengerecht vor und nimmt auch Themen in den Fokus, die bisher als Ursachen der Übergewichtsentwicklung wenig Berücksichtigung fanden, wie z.B. Tagesrhythmik bzw. Schlafmangel, Stress und der Sitzende Lebensstil.

\section{Wissenschaftliche Grundlagen}

Da der Sitzende Lebensstil als ein unabhängiger Risikofaktor der Übergewichtsentwicklung bisher kaum Berücksichtigung fand, verfasste Prof. Gerhard Huber vom Institut für Sport und Sportwissen-

${ }^{1}$ Plattform Ernährung und Bewegung e.V. (peb) schaft der Universität Heidelberg und pebVorstandsmitglied im Auftrag der peb zunächst das Grundlagenpapier „Generations S - Sitzender Lebensstil bei Kindern und Jugendlichen“. Auf dieser Grundlage tauschten sich auf Einladung der peb im Juli 201230 Experten zum Thema aus und steckten den Rahmen für das Handlungsfeld $a b$.

Darauf formierte sich der „peb-Fachbeirat Sitzender Lebensstil“, um die weitere Arbeit von peb in diesem Handlungsfeld inhaltlich zu begleiten:

> Prof. Dr. Gerhard Huber, Universität Heidelberg (Sportwissenschaft)

> Dr. Jens Bucksch, Universität Bielefeld (Gesundheitswissenschaften)

> Uwe Kleinert, peb-Arbeitskreis Kommunikation/Coca Cola (Öffentlichkeitsarbeit)

$>$ Prof. Dr. med. Aloys Berg, M.O.B.I.L.I.S. (Sportmedizin)

> Reinhard Mann, Deutsches Institut für Kinder- und Jugendgesundheit (Psychologie)
> Prof. Dr. Wolf Brettschneider, vormals Universität Paderborn (Sportwissenschaft)

> Prof. Dr. Ingo Froböse, Deutsche Sporthochschule Köln (Sportwissenschaft)

Gemeinsam mit diesem Gremium erarbeitete peb das „Working Paper“, mit dem der wissenschaftliche Hintergrund zum Thema sowie erste Schlussfolgerungen für unterschiedliche Settings auf wenigen Seiten zusammengefasst wurden. Auf dieser Grundlage richtete peb Empfehlungen an Politik und Gesellschaft, um dem Sitzenden Lebensstil entgegenzuwirken und schloss damit die inhaltich-wissenschaftliche Arbeit zunächst ab. Die Papiere, die das inhaltliche Fundament der Arbeit von peb zum Thema bilden, stehen unter dem Stichwort „Sitzender Lebensstil“ unter www.pebonline.de als Download zur Verfügung.

\section{Ansprache Multiplikatoren}

Auf dieser Basis entwickelte peb eine Kommunikationsstrategie, um die für gesellschaftliche Veränderungen unverzichtbaren Multiplikatoren (bzw. Stakeholder) für das Thema zu gewinnen. In diesem Zusammenhang fand im Sommer 2013 die Aktion „Aufstehen! Gegen den Sitzenden Lebensstil“ gemeinsam mit 15 Bundestagsabgeordneten statt. Mit Sitztagebuch und Schrittzähler ausgerüstet begleitete peb die Abgeordneten eine Woche lang und kommunizierte insbesondere über die sozialen Medien. Darüber hinaus platzierte peb Beiträge zum Thema Sitzender Lebensstil in zahlreichen Fachmedien, bei- 
spielsweise in „Frühe Kindheit“ und „Kinder- und Jugendarzt“ sowie „Kinder Praxis“.

Um Schulen für das Thema zu sensibilisieren, entwickelten BildungsCent e.V., ein Impulsgeber, der Schulen bei der Gestaltung von Entwicklungsprozessen und der Einbindung wichtiger gesellschaftlicher Themen in den Schulalltag begleitet, und peb gemeinsam den Ideenwettbewerb „Aufstehen! Weniger Sitzen - mehr Bewegung in Alltag und Schule“. Dadurch konnten zahlreiche Schulen erreicht und über das Thema informiert werden. Die Gewinner des Wettbewerbs wurden im Rahmen der Fachtagung „Aufstehen! Mehr Bewegung und weniger Sitzen im Alltag von Kindern!" ausgezeichnet und erhielten die Gelegenheit, ihre Ideen für weniger Sitzen und mehr Bewegung im Schulalltag dem interessierten Fachpublikum zu präsentieren.

\section{Ansprache der Verbraucher}

Auch für die Zielgruppe der Verbraucher hat die peb Angebote entwickelt, um für den Sitzenden Lebensstil zu sensibilisieren und Verbraucher zum Aufstehen zu motivieren. Für das TV-Format „Peb \& Pebber“, das sich an Vorschulkinder richtet und täglich auf Super RTL ausgestrahlt wird, wurden mehrere Folgen zum Thema entwickelt. Titel wie „Der Steh-auf-und-tanzTanz“, „Die sportlichen Stühle“ oder „Flitzen statt sitzen“ geben bereits einen Eindruck davon, wie die Kinder mit positiven Botschaften zum Aufstehen aufgefordert und an einen gesunden Lebensstil herangeführt werden.
Des Weiteren hat peb einen Sitz-Check für Familien entwickelt. Hier beantworten Eltern alltagsnahe Fragen zum Sitzverhalten in ihrer Familie sowie Wissensfragen zum Sitzen und erfahren am Ende, welchem Sitz-Typ ihre Familie entspricht. Gleichzeitig erhalten sie Empfehlungen zur Veränderung des Sitzverhaltens. Darüber hinaus wurde eine Kinder-Version des Sitz-Checks entwickelt. Der Sitz-Check sowohl in der Familien- als auch in der Kinderversion steht unter dem Schlagwort „Sitzender Lebensstil“ unter www.pebonline.de zur Verfügung und wurde in zahlreiche Portale eingebunden. Nach wie vor besteht für Medien und Akteure der Gesundheitsförderung die Möglichkeit, den Sitz-Check in eigene Online-Angebote einzubinden.

\section{Online zu finden unter http://dx.doi.org/ \\ 10-1055/s-0034-1373868}

Die Plattform Ernährung und Bewegung e.V. (peb) ist ein offenes Bündnis mit über 100 Mitgliedern aus öffentlicher Hand, Wissenschaft, Wirtschaft, Sport, Gesundheitswesen und Zivilgesellschaft. Sie alle setzen sich bei peb aktiv für eine ausgewogene Ernährung und mehr Bewegung als wesentliche Bestandteile eines gesundheitsförderlichen Lebensstils bei Kindern und Jugendlichen ein.

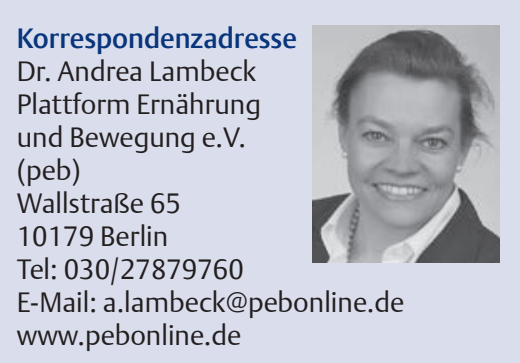

Korrespondenzadresse Dr. Andrea Lambeck Plattform Ernährung und Bewegung e.V. (peb)

Wallstraße 65

10179 Berlin

E-Mail: a.lambeck@pebonline.de www.pebonline.de

\section{Summary}

Peb against sedentary lifestyle

The „Plattform Ernährung und Bewegung e.V. (peb)“ uses „diet and exercise“ to promote health and avoidance of sedentary lifestyle by children and young people. Due to the neglect of sedentary lifestyle as an independent risk factor for children's health, peb's initial step was to develop the basic content of the theme. On this basis, appropriate instruments were developed and implemented in addressing the multipliers and consumers.

Key words: platform, Peb \& Pebber, seat-check, sedentary lifestyle, diet and exercise 